Please quote as: Ebner, W.; Leimeister, J. M. \& Krcmar, H. (2003): Vertrauen in virtuellen Communities: Konzeption und Umsetzung vertrauensunterstützender Komponenten in der Domäne Healthcare. 


\title{
Vertrauen in virtuellen Communities: Konzeption und Umsetzung vertrauens- unterstützender Komponenten in der Domäne Healthcare
}

\author{
Ebner, Winfried; Leimeister, Jan Marco; Krcmar, Helmut \\ Lehrstuhl für Wirtschaftsinformatik (I 17) \\ Boltzmannstr. 3
}

85748 Garching b. München

\begin{abstract}
Zusammenfassung: Vertrauen ist im Umfeld von Online-Anwendungen, insbesondere im Gesundheitsbereich, von zentraler Bedeutung. Vertrauen bildet deshalb (neben anderen Erfolgsfaktoren) die Basis für den erfolgreichen Aufbau und Betrieb einer virtuellen Community (VC). Ein Blick auf bestehende Communities lässt jedoch den Schluss zu, dass in vielen virtuellen Gemeinschaften diesen Anforderungen nicht oder nur unzureichend Rechnung getragen wird. Deshalb beschreibt dieser Beitrag anhand des Falles der Healthcare-Community „,krebsgemeinschaft.de“, wie der bewusste Umgang mit dem Wunsch nach Vertrauen in einer VC konzipiert und umgesetzt werden kann. Die vorgestellten Komponenten unterstützen den Prozess der Vertrauensbildung innerhalb der VC und tragen so entscheidend zum erfolgreichen Aufbau und Betrieb der Gemeinschaft bei. Der Beitrag endet mit einem Ausblick auf noch nicht umgesetzte Aspekte sowie offene Forschungsfragen.
\end{abstract}

Schlüsselworte: Vertrauen, Virtuelle Gemeinschaft, Community, krebsgemeinschaft.de, Healthcare, Wahrgenommene Kompetenz, Wahrgenommenes Wohlwollen, Visitenkarte, Anonymitätskonzept, Afgis Transparenzkriterien.

\section{Einleitung}

Diese Arbeit ist Teil des Forschungsprojekts „COSMOS (Community Online Services and MObile Solutions)“. Dabei handelt es sich um ein Verbundprojekt mehrerer Partner ${ }^{1}$, das sich zum Ziel gesetzt hat, den Aufbau, die Entwicklung und den Betrieb von Virtuellen Communities zu untersuchen.

1 COSMOS ist ein Verbundprojekt der Technischen Universität München, der Universität Hohenheim und $\mathrm{O}_{2}$ Germany (Viag Interkom $\mathrm{GmbH} \& \mathrm{Co}$ ). Das Projekt wird vom 
Ziel der Arbeit ist der Entwurf und die Umsetzung von Komponenten, die dem Wunsch der Mitglieder nach Vertrauen in einer VC gerecht werden. Den Grundstein hierzu legt die Diskussion des Phänomens „Vertrauen“. Zunächst wird sich in Kapitel 1 der Thematik allgemein in Bezug auf Organisationen genähert, um danach abzuleiten, wie sich dieses Konstrukt auf das Umfeld von OnlineAnwendungen und speziell auf die Domäne Healthcare übertragen lässt. In Kapitel 2 werden die vertrauensunterstützenden Komponenten konzipiert, um im folgenden Kapitel 3 eine Auswahl von umgesetzten Komponenten zu präsentieren. In Kapitel 4 werden erste Ergebnisse der umgesetzten Komponenten beschrieben. Der Artikel schließt in Kapitel 5 mit einem Ausblick auf noch ausstehende Aufgaben und Herausforderungen.

\subsection{Grundlegende Überlegungen}

"Als ein elementares Ordnungsprinzip zwischenmenschlicher Austauschbeziehungen spielt Vertrauen (...) eine zentrale Rolle."

$$
\text { [Pico }{ }^{+} \text {01, S. 123] }
$$

Ripperger definiert Vertrauen als die ,freiwillige Erbringung einer riskanten Vorleistung (durch den Vertrauensgeber) unter Verzicht auf explizite vertragliche Sicherungs- und Kontrollmaßnahmen gegen opportunistisches Verhalten (Vertrauenshandlung), in der Erwartung, daß [!] der Vertrauensnehmer freiwillig auf solches Verhalten verzichtet (Vertrauenserwartung)“ [Ripp98, S. 268].

Voraussetzung für die Erfassung der Vertrauensproblematik sind dabei die Phänomene der begrenzten Rationalität und des Opportunismus. Unter begrenzter Rationalität wird der Zustand verstanden, dass der Vertrauensgeber nur unzureichend über die Motive und Handlungsabsichten des Vertrauensnehmers informiert ist. Außerdem kann der Vertrauensnehmer Informationen verzerren oder verheimlichen, um hieraus einen Vorteil gegenüber dem Vertrauensgeber zu erzielen (Opportunismus) [Pico ${ }^{+}$01, S. 125].

Koch et. al. ergänzen, dass in Organisationen sowohl die Koordination der Einzelaktivitäten als auch die Motivation bei der Aufgabenerfüllung zu bewerkstelligen ist. Koordination und Motivation sind risikobehaftet und machen deshalb Vertrauen erforderlich [Koch ${ }^{+} 00$, S. 71].

Diese beiden Aspekte werden durch Abbildung 1 illustriert:

Bundesministerium für Bildung und Forschung, FKZ 01 HW 0107 - 01 HW 0110, gefördert. Für weitergehende Informationen besuchen Sie bitte den Internetauftritt des Verbundprojekts unter http://www.cosmos-community.org. 


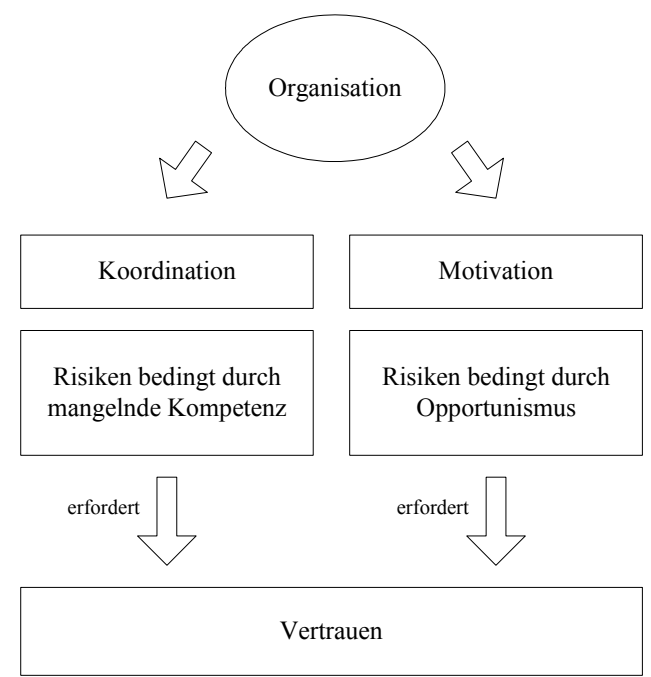

Abbildung 1: Organisation und Vertrauen

Quelle: [Koch ${ }^{+} 00$, S. 71] in Anlehnung an [Wolf00]

Aus sozialwissenschaftlicher Perspektive unterscheidet man drei verschiedene Arten von Vertrauen [AbHa00]:

1. Persönliches Vertrauen: Unter persönlichem Vertrauen wird das direkte Vertrauen zwischen zwei beteiligten Personen verstanden. Dieses Vertrauen ist sowohl personen- als auch kontextspezifisch. Herr Maier vertraut Herrn Müller zum Beispiel in Bezug auf die Beratung bei Finanzanlagen, wohingegen er ihm kein Vertrauen bezüglich der Obhut der eignen Kinder entgegen bringt.

2. Systemvertrauen ${ }^{2}$ : Systemvertrauen ist nicht an die Eigenschaften von Vertrauensgebern gebunden. Es wird durch die wahrgenommen Eigenschaften oder Vertrauenswürdigkeit des Systems oder der Institution bestimmt, in welcher sich Vertrauen entwickeln kann. Als Beispiel sei hierfür das Währungssystem angeführt.

3. Veranlagtes Vertrauen: Das veranlagte Vertrauen beschreibt die generelle Eigenschaft von Vertrauensnehmern, Personen Vertrauen zu schenken. Es handelt sich dabei um eine Art Gundvertrauen, d.h. die für die jeweilige Person grundlegende Einstellung zu sich und seiner Umwelt. Deshalb ist diese Art von Vertrauen weder von Personen noch vom jeweiligen Kontext abhängig.

2 Die Übersetzung lehnt sich bewusst an die Wortwahl Luhmanns an, der im Systemvertrauen ein Einstellung ,jenseits von persönlich geleistetem Vertrauen oder Misstrauen“ sieht [Luhm89, S. 66]. 
Diese Arten von Vertrauen unterscheiden sich zusätzlich dadurch, dass sie verschieden schwer aufzubauen sind. Der Vertrauensaufbau gestaltet sich beim persönlichen Vertrauen noch am leichtesten, wohingegen sich das veranlagte Vertrauen nur schwer beeinflussen lässt. Deswegen wird im Folgenden der Schwerpunkt der Betrachtung auf persönliches Vertrauen und Systemvertrauen gelegt.

\subsection{Rolle von Vertrauen und Reputation im Umfeld von Online-Anwendungen}

Wie schafft man es nun, dieses „Ordnungsprinzip zwischenmenschlicher Austauschbeziehungen“ geplant aufzubauen? Der Weg führt über die Unterscheidung zwischen Vertrauenshandlung, der freiwilligen Erbringung einer riskanten Vorleistung, und Vertrauenserwartung, der Erwartung, dass der Vertrauensnehmer motiviert ist, freiwillig auf opportunistisches Verhalten zu verzichten $\left[\mathrm{Koch}^{+} 00\right.$, S. 72].

Bei einer Vertrauenserwartung setzt man sich über einen Informationsmangel wissentlich hinweg und stützt sich stattdessen auf eigene Erfahrungen und Erfahrungen Dritter. Diese Erfahrungen werden Reputation genannt. Reputation ist demnach die ,erwartete Handlung eines Akteurs, welche auf Informationen über diesen Akteur oder auf Beobachtungen vorangegangen Verhaltens basiert" [AbHa00].

Somit wird Reputation zu einem Indikator, welcher den Aufbau von Vertrauen unterstützt. Abbildung 2 verdeutlicht die Rolle von Reputationsindikatoren:

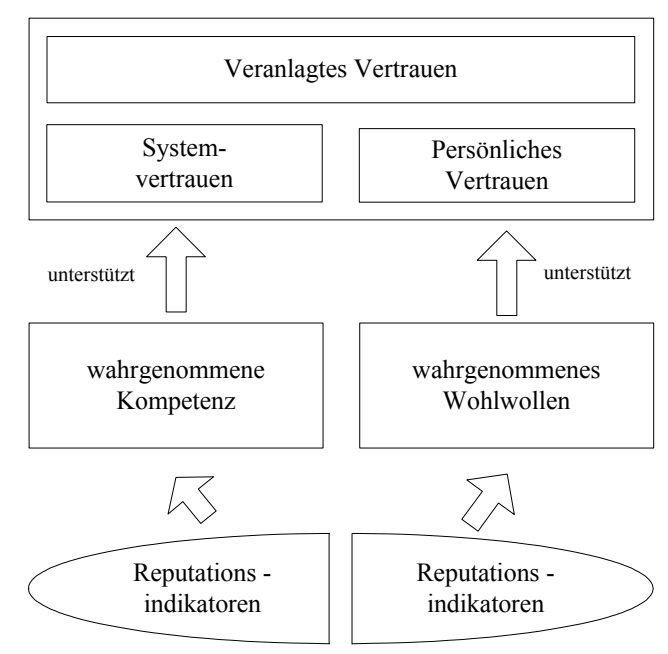

Abbildung 2: Reputationsindikatoren und Vertrauen Quelle: Eigene Darstellung 
Wahrgenommene Kompetenz wird im Offline-Umfeld oft durch Organisationen hergestellt, welche die Reputationsermittlung und -vermittlung übernehmen, beispielsweise die Schutzvereinigung für allgemeine Kreditsicherung (SCHUFA) oder auch Ratingorganisationen für Industrie- und Handelsunternehmen (z.B. EuroRatings AG). Dabei werden Informationen über Transaktionspartner zentral gesammelt und gegen Entgelt zur Verfügung gestellt [Koch ${ }^{+} 00$, S. 75].

Im Online-Umfeld haben sich so genannte Trust Partner etabliert, die als unabhängige Institutionen die Einhaltung von Standards garantieren. Solche Standards werden beispielsweise für sichere Bezahlmethoden und verschlüsselte Datenübertragung gesetzt. Als Beispiele können „Trustet Shops“, „TRUSTe“ oder „Shopinfo"3 genannt werden [Bull ${ }^{+} 02$, S. 235]. Dabei ist jedoch zu beachten, dass diese Institutionen nur die Vertrauensbildung unterstützen können, wenn sie z.B. die Handhabung des Datenschutzes stetig überprüfen und zusätzlich bei Missachtung der Auflagen unter Androhung von Strafen auf die Einhaltung drängen [Schn00, S. 59].

Die wahrgenommene Kompetenz und somit das Systemvertrauen werden zusätzlich durch die klare Definition der unterschiedlichen Verantwortlichkeiten der einzelnen Beteiligten unterstützt. Die Offenlegung von Preisen, Lieferzeiten, Steuern oder Storno-Gebühren schützt den Nutzer vor unangenehmen Überraschungen. Auf der anderen Seite gelten z.B. verbindliche Nutzungsbedingungen und Verhaltensregeln für die Teilnahme an der Gemeinschaft [Schn00, S. 58].

Ein weiterer Reputationsindikator zur Unterstützung der wahrgenommenen Kompetenz ist die Offenlegung von Leistungskennzahlen. So können zum Beispiel Fluggesellschaften die Prozentzahlen ihrer pünktlichen Flüge anbieten oder Einzelhändler Verkaufszahlen bestimmter Produkte. Diese periodischen Berichte und der damit verbundene offene Umgang mit den eigenen erbrachten Leistungen gibt auch skeptischen Nutzern mehr Sicherheiten im Umgang mit der Organisation [Schn00, S. 58].

Das wahrgenommene Wohlwollen (und die damit einhergehende Unterstützung des persönlichen Vertrauens) ist schwieriger zu fassen. Es ist mit dem Erkennen der guten Absichten des Kooperationspartners zu vergleichen. Im Folgenden werden drei Möglichkeiten vorgestellt, welche die Kommunikation und Visualisierung dieser Reputationsindikatoren fördern [Koch ${ }^{+} 00$, S. 75ff.]:

1. Gegenseitige Bewertung von Transaktionspartnern: Viele OnlineAuktionshäuser haben die Möglichkeit geschaffen, dass sich die Beteiligten nach einer Transaktion gegenseitig bewerten. Dabei wird oft eine Mischung

3 Weitere Informationen zur Methodik dieser Gütesiegel finden sich auf den Websites der Organisationen unter www.trustedshops.de, www.truste.org und www.shopinfo.net (zugegriffen am 03.02.03). 
aus einer standardisierten Gesamteinschätzung (z.B. in Form von Sternchen) und einem Feld für Kommentare benutzt. ${ }^{4}$ Problematisch hierbei ist jedoch, dass die Beurteilenden ihre Identität nicht offen legen müssen und somit die Aussagekraft der einzelnen Bewertung eher gering ist. Die Überzeugungskraft dieser Beurteilung liegt eher in der Anzahl der Bewertungen unterschiedlicher Nutzer, denn je mehr Nutzer sich positiv über einen Teilnehmer geäußert haben, je eher ist dieser positiven Bewertung zu trauen [GaWa01, S. 25] .

2. Bewertung von Meinungen: Im Gegensatz zur gängigen Praxis in Auktionshäusern erfolgt hierbei keine direkte Bewertung von Transaktionspartnern. Es werden ausschließlich die Empfehlung bzw. Informationen bewertet, wie dies zum Beispiel bei www.ciao.de mit „sehr nützlich“, „nützlich“, ,,wenig nützlich“ und „nutzlos“ der Fall ist. Außerdem kann der Mittelwert der abgegebenen Bewertungen errechnet und visuell dargestellt werden.

3. Beziehungsnetzwerke: Grundidee dieses Konzepts ist es, einen vertrauenswürdigen Bekannten zu finden, der einem Auskunft über die Vertrauenswürdigkeit eines potentiellen Kooperationspartners gibt. Damit ein System solche Hilfestellungen geben kann, müssen im Vorfeld Informationen über die Kontakte der Besucher untereinander erhoben werden. Die daraus entstehenden Beziehungsnetzwerke werden dann visualisiert ${ }^{6}$.

Besonders bei virtuellen Gemeinschaften besteht die Möglichkeit, Erfahrungen von oder mit Personen einfach zu kommunizieren. Darüber hinaus ist diese Form der Meinungsäußerung mit äußerst geringen Transaktionskosten verbunden.

\subsection{Die Anwendungsdomäne Healthcare - Besonderheiten einer Gemeinschaft für Brustkrebspatienten}

Für den erfolgreichen Aufbau von Vertrauen im Gesundheitsbereich im Allgemeinen und bei der Zielgruppe Krebspatientinnen im Besonderen sind neben den vorgestellten Konzepten vor allem zwei Besonderheiten von entscheidender Bedeutung:

4 Beispiele finden sich bei den verschiedenen Online-Auktionshäuser wie z.B. www.qxl.de, www.ebay.de oder auch bei den zShops von www.amazon.de, bei denen private Verkäufer von Waren vom Käufer bewertet werden können (zugegriffen am 03.02.03).

5 Dass die reine Anzahl an Bewertungen über einen Transaktionspartner nicht alleine ausreicht, um wahrgenommenes Wohlwollen zu unterstützen, zeigen die jüngsten Betrugsfälle bei Online-Auktionshäusern deutlich. Hier ist zukünftig neben der Anzahl der Transaktionen und damit auch der Anzahl der Einzelbewertungen auch der Warenwert der zu Grunde liegenden Transaktionen als Gewichtungsfaktor zu berücksichtigen.

6 Als ein Beispiel für diese Art von Reputationsvermittlung ist ReferralWeb zu nennen (http://www.cs.washington.edu/homes/kautz/referralweb, zugegriffen am 03.02.03). 
Zum einen bedingt die besondere Zielgruppe spezielle Anforderungen an Oberfläche und Aufbau der Plattform ${ }^{7}$. So werden wegen der thematischen Einschränkung auf Brustkrebs vor allem ältere Frauen angesprochen.

Zum anderen ist durch das Thema „Krebs“ als inhaltlicher Schwerpunkt der Gemeinschaft ein Mitwirken der Mitglieder auf besonders persönlicher und intimer Ebene erforderlich. Aus der Sicht der Betroffenen stellt die lebensbedrohliche Diagnose den Anfang einer Phase extremer physischer und psychischer Belastungen dar, deren Ende wegen des möglichen Auftretens von Rezidiven ${ }^{8}$ nicht vorausgesagt werden kann.

Um diese Belastungen bewältigen zu können, benötigen Betroffene und deren Angehörige in allen Stadien der Krankheit umfassende Information. Ganz entscheidend bei diesen überlebenswichtigen Informationen ist dabei die Frage nach der Vertrauenswürdigkeit derselben. ${ }^{9}$ Darüber hinaus geht aus Forschungsarbeiten in Selbsthilfegruppen hervor, dass neben Information als Voraussetzung zur Krisenbewältigung auch weitere Formen der Unterstützung, Training und die physische Verfügbarkeit der Hilfe von Nöten sind, um sich an die neue Situation anzupassen und diese Lebensbedrohung zu verarbeiten [Hase93; LoBr98].

Die Ergebnisse von im Rahmen des Projekts COSMOS durchgeführten Fokusgruppenuntersuchungen verdeutlichen, dass viele der potenziellen Teilnehmer dem Medium Internet zwar interessiert aber dennoch mit Skepsis gegenüber stehen. Besonders die Angst, dass die persönlichen Daten für Werbezwecke missbrauch werden könnten, hemmt die Nutzer in der Anwendung der verschiedenen Dienste [Ebne02].

Es kann also resümiert werden, dass die Mitglieder von KrebspatientenCommunities im Vergleich zu Mitgliedern anderer Communities ein höheres Bedürfnis nach Vertrauen und damit auch höhere Ansprüche den Aufbau von Vertrauen betreffend haben. Diesem erhöhten Bedarf an Vertrauen wird im Folgenden durch die Konzeption vertrauensunterstützender Komponenten für krebsgemeinschaft.de Rechnung getragen.

7 Vgl. hierzu die Konzeption eines Entwicklungsmodells für Healthcare-Communities im Rahmen der Forschungsarbeit [Arno ${ }^{+}$03].

8 Unter „Rezidiven“ wird das Wiederauftreten der Krankheit (=Rückfall) verstanden [Wahr86, S. 1066].

9 Aus Experteninterviews geht hervor, dass die Anzahl von unseriösen und nicht vertrauenswürdigen Angeboten im Internet zur Behandlung von Krebs beträchtlich ist [Ebne02, Anhang XXVII ff.]. 


\section{Konzeption von vertrauensunterstützenden Komponenten}

Die in diesem Kapitel vorgestellten Komponenten stellen konkrete Umsetzungsmöglichkeiten zur Unterstützung des Vertrauensbildungsprozesses dar. Dabei wird nach denjenigen Reputationsindikatoren unterschieden, welche die in Kapitel 1.2 ausdifferenzierten Vertrauenstypen Systemvertrauen und persönliches Vertrauen unterstützen. Die Umsetzung erfolgt für die Community krebsgemeinschaft.de.

\subsection{Wahrgenommene Kompetenz}

Die für die wahrgenommene Kompetenz entscheidenden Komponenten sind der zentral bereitgestellte, qualitätsgesicherte Inhalt, das Betreibermodell und das Rechtekonzept, das den Zugang zu verschiedenen Funktionalitäten je nach Benutzertyp regelt.

\subsubsection{Transparenzkriterien des Aktionsforums Gesundheits- informationssysteme (afgis)}

Um die „wahrgenommene Kompetenz“ zu erhöhen, helfen wie dargelegt unabhängige Institutionen, welche die Einhaltung von Standards überprüfen können.

Im deutschsprachigen Raum hat sich dafür 1999 das Aktionsforum Gesundheitsinformationssystem (afgis) gebildet, das die Entwicklung und Etablierung eines dauerhaften Qualitätssicherungsprozesses für Gesundheitsinformation im Internet vorantreibt. Um dies zu erreichen, wurden von der Arbeitsgruppe „Qualitätssicherung/Qualitätsmanagement" in einem ersten Schritt folgende zehn Transparenzkriterien ausgearbeitet $[\text { Moel02 }]^{10}$ :

- Transparenz über die Anbieter

- Transparenz über Ziel, Zweck und angesprochene Zielgruppe(n) der Information

- Transparenz über die Autoren und die Datenquellen der Information

- Transparenz über die Aktualität der Daten

- Möglichkeit für Rückmeldungen seitens der Nutzer

- Transparenz über Verfahren der Qualitätssicherung

${ }^{10}$ Eine detailliert Beschreibung der zehn Transparenzkriterien kann online unter http://www.afgis.de/qualitaetssicherung.php eingesehen werden (zugegriffen am 03.02.03). 
- Transparente Trennung von Werbung und redaktionellem Beitrag

- Transparenz über Finanzierung und Sponsoren

- Transparenz über Kooperationen und Vernetzung

- Transparenz über Datenverwendung und Datenschutz.

Die Operationalisierung dieser Kriterien ist aktuell Gegenstand der Arbeit von afgis.

\subsubsection{Qualitätsgesicherter Inhalt}

Eine weitere Unterstützung der „wahrgenommenen Kompetenz“ bildet der qualitätsgesicherte Inhalt. Hierzu wird für den Fall von krebsgemeinschaft.de qualitätsgesicherter Inhalt vom anerkannten Krebsinformationsdienst (KID) am Krebsforschungszentrum in Heidelberg eigens für den Aufbau der Plattform erstellt. Erfahrungen, die seit 1996 bei der telefonischen Beratung und auch bei Anfragen per E-Mail gesammelt worden sind, gehen zusammen mit den neuesten Forschungsergebnissen in die Produktion der Texte ein. Zur Qualitätssicherung werden die Texte von zwei verschiedenen anerkannten Experten des Bereichs Onkologie gegengelesen und gegebenenfalls korrigiert.

\subsubsection{Betreibermodell von krebsgemeinschaft.de}

Die Motivation und der Hintergrund der Betreiber einer virtuellen Community spielt für die wahrgenommene Kompetenz eine zentrale Rolle. So wird ein Patient in einer Community für Krebspatienten, die von einem Pharma-Konzern betrieben wird, der Medikamente für Chemotherapien herstellt, weniger Kompetenz bspw. im Bereich alternativer Therapien vermuten als dies bei einem Institutionen übergreifenden nicht-kommerziellen Betreiber-Verbund der Fall ist.

Aus diesem Grunde ist die Offenlegung von Informationen zum Betreibermodell eine wichtige Stütze für den Aufbau von Vertrauen in einer VC.

\subsubsection{Rechtekonzept}

Wie in Kapitel 1.3 dargelegt, ist neben der Unterstützung durch Information vor allem die Interaktion als Hilfestellung für die Betroffenen von hoher Bedeutung. Aus diesem Grunde wurden die Austauschdienste Diskussionsforum, Fragen an Experten, Kontaktsuche und Chat nebst einem nach Nutzertyp unterscheidenden Rechtekonzept für alle Funktionalitäten entworfen und realisiert.

Das Diskussionsforum unterstützt hierbei vor allem den asynchronen Austausch von Informationen zwischen den Mitgliedern der Gemeinschaft. Die Fragen an Experten stellen eine spezielle Form des Diskussionsforums dar, weil nur während eines bestimmtes Zeitraums Fragen an einen Experten (vor allem Ärzte mit Spezi- 
alwissen) gestellt werden können, die dann auch nur vom Experten selbst beantwortet werden. Der Austauschdienst Kontaktsuche dient den Mitgliedern der Gemeinschaft als Unterstützung bei der Suche nach Menschen, die sich in einer ähnlichen Lebenslage befinden oder ähnliche Interessen haben. Der Austauschdienst Chat bietet den Mitgliedern der Gemeinschaft die Möglichkeit, sich ohne große Mühe in Echtzeit mit anderen Betroffenen über diejenigen Themen austauschen zu können, die sich gerade beschäftigen. ${ }^{11}$

Das Rechtekonzept regelt den Zugang und die Nutzung der beschriebenen Austauschdienste. Dabei orientiert es sich an den fünf verschiedenen sozialen Rollen, die durch Kim innerhalb ihrer Studien zum Aufbau von Communities identifiziert wurden. Kim unterscheidet dabei zwischen Besucher, Neuling, Mitglied, Leader und Senior [Kim01, S. 134 f.]. Den sozialen Rollen entsprechend werden zunächst drei Rechtestufen vergeben. Der einfache Besucher hat die Rechtestufe Gast, mit der Registrierung erhält der Benutzer die Rechtestufe Mitglied. Im Laufe seiner Mitgliedschaft kann ein Benutzer die Rechtestufe VIP-Mitglied erhalten.

Zusätzlich werden innerhalb von krebsgemeinschaft.de wegen der domänenspezifischen Besonderheiten sowie dem Betreibermodell die Rechtestufe Experte sowie unterschiedliche Administratorenrechte vergeben. So ergeben sich zum Beispiel die Rechtestufen Communitymanager und Contentmanager: Während ein Arzt des Onkologischen Schwerpunkts Stuttgart für die Betreuung der Community zuständig ist (Communitymanager), pflegt der Krebsinformationsdienst am Krebsforschungszentrum in Heidelberg die Informationsseiten von krebsgemeinschaft.de (Contentmanager).

Aus diesem Grund gibt es innerhalb der Gemeinschaft sieben Rechtestufen:

\begin{tabular}{|l|l|l|}
\hline Nr. & Rechtestufe & Beschreibung / Weg zur Rechtestufe \\
\hline 1 & Gast & Unregistrierte Benutzer der Seiten \\
\hline 2 & Mitglied & Registrierter Nutzer \\
\hline 3 & VIP-Mitglied & $\begin{array}{l}\text { Registrierter Nutzer mit besonderen Rechten - } \\
\text { wird durch den Communitymanager von Mitglied-Rechtestufe } \\
\text { aufgewertet (permanent) oder erhält Rechte innerhalb des Chats } \\
\text { von anderem VIP-Mitglied (temporär). }\end{array}$ \\
\hline 4 & Experte & $\begin{array}{l}\text { Fachlicher Experte - } \\
\text { per Ernennung durch Communitymanager, besondere Rechte für } \\
\text { sein „,Thema der Woche“, ansonsten wie Rechtestufe Mitglied. }\end{array}$ \\
\hline Nr. & Rechtestufe & Beschreibung / Weg zur Rechtestufe \\
\hline
\end{tabular}

11 Die konkrete viuselle Umsetzung der Austauschdienste kann unter http://www.krebsgemeinschaft.de eingesehen werden (zugegriffen am 03.02.03). 


\begin{tabular}{|l|l|l|}
\hline 5 & $\begin{array}{l}\text { Community- } \\
\text { manager }\end{array}$ & $\begin{array}{l}\text { Betreuung der Gemeinschaft (z.B. Beantwortung von Mit- } \\
\text { gliederanfragen und Rekrutierung von Experten)- } \\
\text { per se durch Projektteam }{ }^{12} \text { bestimmt. }\end{array}$ \\
\hline 6 & $\begin{array}{l}\text { Content- } \\
\text { manager }\end{array}$ & $\begin{array}{l}\text { Inhaltliche Verantwortung und Pflege der Informationsseiten - } \\
\text { per se durch Projektteam bestimmt. }\end{array}$ \\
\hline 7 & Administrator & $\begin{array}{l}\text { Technische Administration der Plattform - } \\
\text { per se durch Projektteam bestimmt. }\end{array}$ \\
\hline
\end{tabular}

Tabelle 1: Rechtestufen von krebsgemeinschaft.de Quelle: Eigene Darstellung

Durch diese verschiedenen Rechtestufen werden Rechte auf die einzelnen Funktionalitäten der Dienste von krebsgemeinschaft.de verteilt. Die wichtigsten Eckpunkte des Rechtekonzepts sind zusammengefasst die folgenden ${ }^{13}$ :

- Unregistrierte Benutzer der Plattform (Gäste) können bei den beiden Austauschdiensten Diskussionsforum und Fragen an Experten jeweils die Übersichtsseite sehen, allerdings keine Beiträge betrachten oder erstellen. Der Zugriff auf die Kontaktsuche und den Chat bleibt unregistrierten Benutzern verwehrt.

- Die Funktionalität Beitrag/Frage ändern von Diskussionsforum und Fragen an Experten ist sowohl für Mitglieder als auch VIP-Mitglieder auf eigene Beiträge bzw. Fragen beschränkt. Die eigene Frage kann in den Fragen an Experten nur so lange verändert werden, bis der Experte auf sie geantwortet hat.

- Experten und der Administration (Rechtestufe 5-7) ist es möglich, auch Beiträge anderer Mitglieder zu ändern und somit bei Verletzungen der Forenregeln die Beiträge zu kommentieren bzw. diese auch zu löschen (Rechtestufe 5 und 7).

- In der Ausbaustufe des Chats hat jedes Mitglied die Möglichkeit, ein temporäres VIP-Mitglied zu werden. Dies geschieht dadurch, dass das Mitglied mit der Funktionalität ,Raum eröffnen“ einen neuen Chatraum eröffnet und dann in diesem Raum (und nur in diesem eigenen Raum) VIP-Mitglied-Rechte hat. Diese Recht gelten dann nur für den Chat und nur so lange das Mitglied in diesem Chatraum ist.

\subsection{Wahrgenommenes Wohlwollen}

Das „wahrgenommene Wohlwollen“ kann sich einerseits auf die Betreiber einer VC beziehen, andererseits auf die anderen Mitglieder der Gemeinschaft. Um den

12 Das Projektteam setzt sich aus Mitarbeitern der beteiligten Projektpartnern zusammen.

13 Eine detaillierte Zuordnung der verschiedenen Rechte auf die einzelnen Funktionalitäten der Dienste von krebsgemeinschaft.de kann bei den Autoren angefordert werden. 
Mitgliedern von krebsgemeinschaft.de größtmögliches Wohlwollen von Seiten der Betreiber zu signalisieren, wird im Rahmen des Betreibermodells aktiv auf die Motivation der beteiligten institutionellen Partner und das Fehlen von kommerziellen Interessen hingewiesen. Das „wahrgenommene Wohlwollen“ der Nutzer der Gemeinschaft untereinander wird durch die Bereitschaft der Mitglieder unterstützt, aus der völligen Anonymität herauszutreten und anderen Teilnehmern die Möglichkeit zu geben, mehr über die eigene Person zu erfahren.

\subsubsection{Visitenkarte}

Die Visitenkarte bietet zur Offenlegung des eigenen Wohlwollens gegenüber anderen Nutzern eine gute Möglichkeit. Sie beinhaltet Informationen, die sich aus Registrierungsdaten ${ }^{14}$ und weiteren optionalen Daten zusammensetzen. Immer dann, wenn ein Teilnehmer innerhalb der Gemeinschaft einen Beitrag veröffentlicht oder eine Frage stellt, wird sein Benutzername als Hyperlink angezeigt. Durch einen Klick auf diesen Link gelangt man auf die Visitenkarte des Nutzers, auf der, abhängig vom Anonymitätsgrad, persönliche Informationen zu finden sind. Außerdem haben die Daten der Visitenkarte eine weitere Funktion: Durch die Angabe von persönlichen Informationen ist es Mitgliedern mit Hilfe der Kontaktsuche möglich, andere Mitglieder zu finden.

Tabelle 2 beschreibt die optionalen Felder der Visitenkarte:

\begin{tabular}{|c|c|c|}
\hline Ref. & Feldname & Bemerkungen \\
\hline \multicolumn{3}{|c|}{ Persönliche Daten: } \\
\hline /D05/ & Anrede & Werte: „Herr“, „Frau“ \\
\hline /D15/ & Titel & Format: freies Textfeld \\
\hline /D130/ & Geburtstag & Format: „TT.MM.JJJJ““ \\
\hline /D140/ & Familienstand & $\begin{array}{l}\text { Werte: „keine Angabe “"15 } \\
\text { schieden“, „ledig“, ,getrennt lebend“, ,, ,in Lerheiratet", ,ge- } \\
\text { lebend“ und ,verwitwet“" } \\
\text { - Format: Auswahlliste }\end{array}$ \\
\hline /D150/ & Eigene Kinder & $\begin{array}{l}\text { Werte: ,keine Angabe“" , „nein“, ,ja“" } \\
\text { - Format: Radio Buttons }\end{array}$ \\
\hline /D160/ & Bild & Format: JPEG oder GIF \\
\hline
\end{tabular}

14 Als Registrierungsdaten sind obligatorisch Benutzername, Vorname, Nachname, EMail, Passwort, Anonymitätsgrad und vollständige Adresse anzugeben.

15 Der vorselektierte Wert (Standard) wird unterstrichen dargestellt. 


\begin{tabular}{|c|c|c|}
\hline Ref. & Feldname & Bemerkungen \\
\hline$/ \mathrm{D} 170 /$ & Eigene Homepage & Format: URL \\
\hline /D180/ & Telefon Mobil & Format: freies Textfeld \\
\hline /D190/ & Telefon privat & Format: freies Textfeld \\
\hline$/ \mathrm{D} 200 /$ & Fax & Format: freies Textfeld \\
\hline \multicolumn{3}{|c|}{ Die Krankheit: } \\
\hline$/ \mathrm{D} 210 /$ & $\begin{array}{l}\text { Verbindung zur } \\
\text { Krankheit }\end{array}$ & $\begin{array}{l}\text { Werte: „Betroffene } / \mathbf{r}^{\prime \prime},, \text { Angehörige/r“, „Experte/in“, } \\
\text { „Sonstiges“ }\end{array}$ \\
\hline$/ \mathrm{D} 220 /$ & Verbindung (sonst.) & Format: Freies Textfeld \\
\hline$/ \mathrm{D} 230 /$ & Diagnosedatum & Format: „TT.MM.JJJJ“ \\
\hline$/ \mathrm{D} 240 /$ & Krebsart & $\begin{array}{l}\text { Werte: „Brustkrebs“, „Leukämie“, „Sonstiges“, „,keine } \\
\text { Angaben“ - Format: Auswahlliste }\end{array}$ \\
\hline$/ \mathrm{D} 250 /$ & Krebsart (sonst.) & Format: freies Textfeld \\
\hline /D260/ & Krankheitsstadium & $\begin{array}{l}\text { Werte: „,keine Angabe“, „Vor Therapiebeginn“, „Unter } \\
\text { Therapie“, „Geheilt"“, „Keine Krebsansiedlungen (Me- } \\
\text { tastasen)“, „Krebsansiedelungen (Metastasen)“ - } \\
\text { Format: Auswahlliste }\end{array}$ \\
\hline$/ \mathrm{D} 270 /$ & Therapieform & $\begin{array}{l}\text { Werte: „Radikale Operation (Brustentfernung)“, „Strah- } \\
\text { lentherapie“, „Lymphknotenentfernung“" } \\
\text { „brusterhaltende Operation“, „Hormontherapie“, „,Wie- } \\
\text { deraufbau der Brust““, „Chemotherapie“, } \\
\text { „Antikörpertherapie““, „Alternativtherapie“ - Format: } \\
\text { Checkboxes mit Möglichkeit der Mehrfachauswahl }\end{array}$ \\
\hline /D280/ & Therapieform (sonst.) & Format: freies Textfeld \\
\hline \multicolumn{3}{|c|}{ Persönliche Interessen: } \\
\hline /D290/ & Freizeitaktivitäten & $\begin{array}{l}\text { Werte: „Musik“, „,Oper/Theater/Musical“, „Tanzen“, } \\
\text { „Spaziergänge“, „Kino““, ,gesellschaftliches und sozia- } \\
\text { les Engagement“, „,Reisen“, „Kunst/Ausstellungen“ - } \\
\text { Format: Checkboxes mit Möglichkeit der Mehrfach- } \\
\text { auswahl }\end{array}$ \\
\hline /D300/ & Interessen (sonst.) & Format: freies Textfeld \\
\hline
\end{tabular}

Tabelle 2: Optionale Daten der Visitenkarte

Quelle: Eigene Darstellung

\subsubsection{Anonymitätskonzept}

Wie zuvor ausgeführt entscheidet jedes Mitglied selbst darüber, welche Daten es anderen Mitgliedern der Gemeinschaft zeigen will. Damit wird der Problematik Rechnung getragen, dass jede Person individuell mit ihrer Krankheit umgeht und deshalb auch unterschiedlich anonym den Mitgliedern der Gemeinschaft gegenüber auftreten will. Dabei kann jedes Mitglied zwischen vier verschiedenen Anonymitätsgraden wählen. 
Tabelle 3 verdeutlicht die Auswirkungen, die durch die Wahl der verschiedenen Anonymitätsgrade entstehen:

\begin{tabular}{|l|l|}
\hline Anonymitätsgrad & Auswirkungen auf die Darstellung der Visitenkarte \\
\hline Nichts anzeigen & Anzeige: ,Der Benutzer möchte seine Daten nicht anzeigen!“ \\
\hline Anonymisiert & $\begin{array}{l}\text { Angezeigt werden: Benutzername, Bundesland, Nutzerstatus, } \\
\text { Verbindung zur Krankheit, Diagnosedatum, Krebsart, Krebs- } \\
\text { stadium, Therapieform, Freizeitaktivitäten, Interessen } \\
\text { (sonstiges) }\end{array}$ \\
\hline $\begin{array}{l}\text { Alle Anonymisiert - } \\
\text { Freunde Alles }\end{array}$ & $\begin{array}{l}\text { Mitgliedern werden angezeigt: Benutzername, Bundesland, } \\
\text { Nutzerstatus, Interessen, Verbindung zur Krankheit, Diagnose- } \\
\text { datum, Krebsart, Krebsstadium, Therapieform, } \\
\text { Freizeitaktivitäten, Interessen (sonstiges) } \\
\rightarrow \text { Freunden werden alle eingegebenen Daten angezeigt }\end{array}$ \\
\hline Alles anzeigen & Angezeigt werden: Alle eingegebenen Daten \\
\hline
\end{tabular}

Tabelle 3: Anonymitätsgrade und deren Auswirkung auf die Darstellung der Visitenkarte Quelle: Eigene Darstellung

Der Anonymitätsgrad „Alle Anonymisiert - Freunde Alles“ bietet den Mitgliedern eine besondere Differenzierung. Wenn sich zu einem Teilnehmer der Gemeinschaft z.B. durch einen Chat oder persönliche Bekanntschaft schon ein Vertrauensverhältnis aufgebaut hat, so kann man diesen durch Eingabe des Benutzernamens in eine Freundesliste aufnehmen. Dieser Freund hat dann die Möglichkeit, alle eingegebenen Daten der Visitenkarte einzusehen, wohingegen normale Mitglieder nur die anonymisierte Form der Visitenkarte dargestellt bekommen.

\section{Ausgewählte Beispiele der Umsetzung von Komponenten anhand des Falles www.krebsgemeinschaft.de}

Nach Darstellung des Soll-Konzeptes zur Unterstützung des Aufbaus von Vertrauen in VCs erfolgt nun die Darstellung der Umsetzung ausgewählter Komponenten. Hierzu wird zunächst beschreibend auf die Umsetzung der Afgis Qualitätskriterien eingegangen. Sie sollen das Systemvertrauen der Mitglieder stärken. Der Schwerpunkt der danach folgenden Darstellung liegt auf der Visitenkarte sowie den Anonymitätsgraden und damit denjenigen Komponenten, die das Persönliche Vertrauen aufbauen. 


\subsection{Umsetzung: Wahrgenommene Kompetenz}

Die Afgis Qualitätskriterien wurden folgendermaßen auf krebsgemeinschaft.de umgesetzt:

Die Transparenz über die Anbieter gewährleistet das Impressum, das unter dem Link ,Über uns' zu finden ist. Außerdem ist auf jeder Seite das Logo als visuelle Markierung zu sehen. Die Transparenz über Ziel, Zweck und angesprochene Zielgruppe wird schon durch die Wahl der URL sichtbar: www.krebsgemeinschaft.de. Auf der Startseite findet sich darüber hinaus ein Statement über die Fokussierung der Plattform auf Brustkrebserkrankte, Angehörige und Interessierte. Hinter dem Link ,Geführte Tour' werden alle Bereiche der Plattform vorgestellt. Das Redaktionssystem gewährleistet die Ausstattung jedes Beitrags mit einem Aktualisierungsvermerk. Zusammen mit der mit Contentmanager KID wird so der Transparenz über die Autoren und die Datenquellen sowie der Transparenz über die Aktualität der Daten Rechnung getragen.

Die Möglichkeiten für Rückmeldungen seitens der Nutzer sind vielfältig. Zum einen können die Benutzer mit dem Link ,Anregungen und Kritik' ihre Anmerkungen zur Gestaltung der Seite und den Inhalten geben. Zum anderen besteht die Möglichkeit, durch den Verweis ,Kontakt' die Community Manager direkt mit einer Email zu kontaktieren. Der Verweis ,Über uns' enthält weitere Kontaktmöglichkeiten der Projektpartner.

Komplexer ist die zu gewährleistende Transparenz über Verfahren der Qualitätssicherung. Durch die Organisation der Inhalte in einzelne Items, die jeweils mit Zusatzinformationen zu Datum, zugeordneter Kategorie und Autor versehen sind, wird ersichtlich, wer für die Qualität des Beitrags zuständig ist. Außerdem sind Informationen, die inhaltlich qualitätsgeprüft sind, deutlich durch die Navigation von Meinungen oder nicht qualitätsgesicherter Information (z.B. im Diskussionsforum) getrennt. Weitere deutliche Hinweise in Textform weisen den Benutzer bei den Austauschdiensten darauf hin, dass die Mitglieder von krebsgemeinschaft.de für ihre eigenen Beiträge selbst verantwortlich sind und für die Richtigkeit der Aussagen von Betreiberseite her keine Verantwortung übernommen werden kann .

Die Forderung von afgis nach transparenter Trennung von Werbung und redaktionellem Beitrag trifft für diese Gemeinschaft nicht $\mathrm{zu}$, da sie sich nicht über Werbung finanziert. Transparenz über Finanzierung und Sponsoren ist gegeben, da die Startseite über das Projekt, (fördernde) Projektpartner und die Förderung durch das Bundesministerium für Bildung und Forschung Auskunft gibt. Des Weiteren verweist die Seite „Über uns“ auf die Webseiten der Projektpartner, die ihrerseits ausführliche Informationen bereitstellen. Dies dient der Transparenz über Kooperationen und Vernetzung. Während der Registrierung werden die Teilnehmer über die Nutzungsbedingungen und mit der Datenschutzerklärung über die Verwendung ihrer personenbezogenen Daten aufgeklärt. Somit ist die Forderung nach einer transparenten Datenverwendung und Datenschutz erfüllt. 


\subsection{Umsetzung: Wahrgenommenes Wohlwollen}

Die Visitenkarte und das Anonymitätskonzept wurden in eingeschränkter Form umgesetzt. Einige der konzipierten Felder sind noch nicht in die Visitenkarte aufgenommen worden.

Mit der Umsetzung bietet die Visitenkarte den Mitgliedern von krebsgemeinschaft.de die Möglichkeit, sich untereinander besser kennen zu lernen. Aus Anregungen aus dem laufenden Betrieb heraus wurde eine weitere Funktionalität der Visitenkarte hinzugefügt. Den Mitgliedern ist es nun möglich, dem angezeigten Mitglied über einen Link unter der Visitenkarte eine E-Mail zu schreiben und so sofort persönlich mit ihm in Kontakt zu treten.

Abbildung 3 gibt einen Eindruck der Visitenkarte und deren angezeigte Daten im Anonymitätsgrad „Alles Anzeigen“:

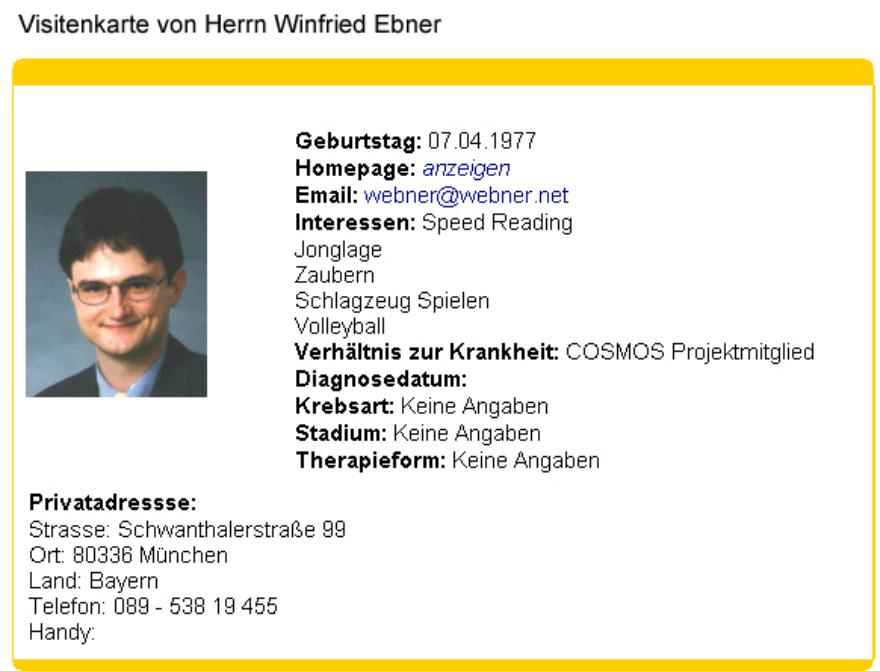

Dem Mitglied pu eine persönliche Email $\equiv$ schreiben

Abbildung 3: Visitenkarte

Quelle: http://www.krebsgemeinschaft.de, zugegriffen 04.02.03

\section{Erste Ergebnisse}

Die Evaluation der vertrauensunterstützenden Komponenten steht zum Zeitpunkt der Beitragserstellung erst am Anfang. Mit den Methoden der Befragung, der Beobachtung und der Dokumentenanalyse werden die verschiedenen Komponenten 
im weiteren Verlauf des Projekts untersucht. Erste vorläufige Ergebnisse sind durch die Dokumentenanalyse der Diskussionsforen vorhanden [Ebne02]. Die folgenden Beiträge aus dem Diskussionsforum sollen exemplarisch verdeutlichen, wie die Mitglieder von krebsgemeinschaft.de miteinander interagieren und mit welcher Offenheit und Einfühlungsvermögen die Mitglieder miteinander umgehen.

Preece hat in ihren Studien über das Erleben von Empathie ${ }^{16}$ in VC herausgefunden, dass dieses Phänomen vor allem in Gemeinschaften auftritt, die themenbezogene Unterstützung bieten [PrGh01, S. 254]. Krebsgemeinschaft.de ist genau zu einer solchen „Support-Community“ zu zählen, auf der neben der reinen Information auch emotionale Unterstützung zu finden ist.

Abbildung 4 verdeutlicht innerhalb eines Beitrags im Diskussionsforum diese starke emotionale Bindung:

\begin{tabular}{|c|c|c|}
\hline Autor & Thema & \\
\hline \multirow[t]{2}{*}{ "orivast } & $\begin{array}{l}20.01 .0322: 05 \\
\text { Ich habe ein halbes Jahr Chemo geschafft!!! Ich } \\
\text { kann euch gar nicht sagen wie erleichtert ich bin :- } \\
\text { ) Jetzt freu ich mich auf den Sport den ich wieder } \\
\text { extrem treiben werde, auf die Harre die wieder } \\
\text { nachwachsen werden, auf die Lebensenergie, die } \\
\text { wieder in meinen Körper strömen wird, auf das } \\
\text { Arbeiten, welches mir so gefehlt hat und auf die } \\
\text { Partys, auf die ich wieder gehen werde. Ich fühl } \\
\text { mich wirklich wie ein neuer Mensch, obwohl die } \\
\text { Angst, dass ich wieder krank werden könnte jetzt } \\
\text { größer ist als je zuvor! Aber wie hat Lx's: : ': } \ddot{i} \\
\text { immer gesagt: wir müßen nach vorne schauen } \\
\text { und WiR bleiben gesund. Ich grüße auch ganz lieb } \\
\text { alle die mich kennen und ich hoffe wir treffen uns } \\
\text { jetzt endlich mal wieder im Chat!!!???? }\end{array}$ & antworten \\
\hline & Reaktionen & \\
\hline 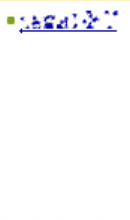 & $\begin{array}{l}21.01 .0311: 41 \\
\text { oh mensch za } * * \cdot ! \text { ich bin so froh das es dir } \\
\text { wieder gut geht! du hast es geschafft!!!!!!! weil } \\
\text { du gekämpft hast und weil du es wolltest. und zum } \\
\text { thema chat: " du hast ja nie zeit" *fg wir würden } \\
\text { uns alle freuen dich bald mal wieder im chat zu } \\
\text { treffen!! (irgendwie vermisse ich deine frechen } \\
\text { sprüche) * smile lieben gruß } . * 2 .\end{array}$ & antworten \\
\hline Seite 1 & & blättern \\
\hline
\end{tabular}

Abbildung 4: Beitrag im Diskussionsforum (1)

Quelle: http://www.krebsgemeinschaft.de, zugegriffen 04.02.03

In weiteren Beiträgen zeigt sich, wie schnell Mitglieder auf die persönliche Ebene kommen und auf ihre Lebenssituation eingehen, um möglichst genau darzustellen,

${ }^{16}$ Empathie ist die Fähigkeit, sich in die Situation und Gefühle einer anderen Person hineinversetzen zu können und sich so mit ihr zu identifizieren [PrGh01, S. 237]. 
in welcher Lage sie sich gerade befinden. Der nächste Beitrag steht am Ende einer Diskussion über das Thema „Deprimiertheit“ und verdeutlicht, dass innerhalb der Gemeinschaft genügend Vertrauen aufgebaut wurde, um auch Tabuthemen zu diskutieren:

\begin{tabular}{|c|c|c|}
\hline - ckross & $\begin{array}{l}15.10 .0211: 13 \\
\text { Hallo Egsastal Meine diagnose war 01/02. Bei mir } \\
\text { konnte zwar brusterhaltend operiert werden, mit } \\
\text { Depressionen, Panikattacken und Angstzuständen } \\
\text { habe ich aber auch zu kämpfen. Jeder (Ärzte) } \\
\text { sagt, das kommt vom Tamoxifen und Zoladex. } \\
\text { Aber es ist beruhigend zu erfahren, dass es } \\
\text { Leidensgenossinen tatsächlich so geht. Ich dachte } \\
\text { auch immer, dass ich das alles alleine schaffe, } \\
\text { aber je mehr Zeit vergeht, je schwerer fällt es mir. } \\
\text { Grüße }\end{array}$ & antworten \\
\hline - & 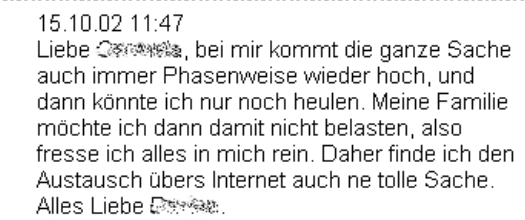 & antworten \\
\hline Seite 1 & & blättern \\
\hline
\end{tabular}

Abbildung 5: Beitrag im Diskussionsforum (2)

Quelle: http://www.krebsgemeinschaft.de, zugegriffen 01.11 .02

Man kann aus den Beiträgen der Mitglieder von krebsgemeinschaft.de deshalb folgern, dass genügend Vertrauen vorhanden ist, um mit Empathie innerhalb der Gemeinschaft miteinander zu kommunizieren.

Da eine Operationalisierung des mehrdimensionalen Konstruktes Vertrauen innerhalb des Projektes noch nicht vorgenommen wurde, ist z.B. eine genaue Zuordnung von vertrauensunterstützenden Komponenten $\mathrm{zu}$ einem möglichen Vertrauenszuwachs noch nicht möglich.

Weitere Ergebnisse bezüglich der Vertrauenswürdigkeit der Gemeinschaft und dem gegenseitigen Vertrauen der Mitglieder werden im Rahmen einer Mitgliederbefragung erwartet.

\section{Ausblick}

Obwohl das Leben in der VC krebsgemeinschaft.de für ein intaktes Vertrauensverhältnis unter den Mitgliedern und zwischen Mitgliedern und Betreibern spricht, sind bei weitem noch nicht alle Möglichkeiten der technischen und organisatorischen Unterstützung von Vertrauen ausgeschöpft. Neben der Umsetzung der noch 
fehlenden Aspekte aus den zuvor dargestellten Komponenten gibt es noch weitere Komponenten.

Eine weitere Unterstützung des ,wahrgenommenen Wohlwollens“ durch die Mitglieder könnte die Bewertung von Informationen sein. Hierbei geht es um die Bewertung einzelner Informationseinheiten (Items), die von jedem registrierten Nutzer durchgeführt werden kann. So könnte neben den Qualitätssicherungsmaßnahmen von Anbieterseite (siehe Kapitel 2.1.2) auch festgestellt werden, ob für Mitglieder der Gemeinschaft die Texte informativ und lesenswert oder unnützlich gewesen sind. Außerdem verstärkt sich durch positive Reaktion auf inhaltliche Beiträge das Vertrauen in die Qualität der angebotenen Texte. Ebenso ist eine Bewertung der von den Nutzern erstellten Inhalten (bspw. einzelne Forumsbeiträge, etc.) möglich, wodurch insbesondere das Vertrauen zwischen den Mitgliedern weiter unterstützt werden kann.

Neben den technischen Unterstützungsmöglichkeiten für den Aufbau und Erhalt von Vertrauen ist auch die Rolle des Community-Managements zentral. Eine nach intersubjektiv nachvollziehbaren Regeln ablaufende, inhaltlich fundierte und im Dienst der Gemeinschaft stehende Moderation ist eine zentrale Einflussgröße auf das Vertrauen in VCs. Wie diese Regeln für die Moderation auszusehen haben, wann auf welche Art was moderiert wird, ist ebenfalls nur ansatzweise erforscht.

Im weiteren Forschungsverlauf stellt sich zudem die Frage, wie ein Geschäfts- und Betreibermodell für Healthcare-Communities außerhalb eines öffentlich geförderten Forschungsprojektes aussehen kann. Denn nur eine sozial akzeptierte, technische stabile und ökonomisch sinnvolle Lösung gewährleistet den Erfolg einer virtuellen Gemeinschaft für Krebspatienten.

\section{Literatur}

[AbHa00] Abdul-Rahman, A.; Hailes, S.: Supporting Trust in Virtual Communities. In: Proceedings of the 33rd Hawaii International Conference on System Sciences (HICSS 33), Maui, Hawaii, January 4-7, 2000.

[Arno ${ }^{+}$03] Arnold, Y.; Leimeister, J.M., Krcmar, H.: COPEP: A Development Process Model for a Community Platform for Cancer Patients. In: Proceedings of the XIth European Conference on Information Systems (ECIS), 2003, Napoli/Naples.

[Bull ${ }^{+}$02] Bullinger, H.-J.; Baumann, T.; Fröschle, N.; Mack, O.; Trunzer, T.: Business Communities. Galileo Press, Bonn 2002.

[Ebne02] Ebner, W.: Community Building: Bedarfsgerechte Entwicklung und Einführung einer virtuellen Gemeinschaft für Krebspatienten. Diplomarbeit am Lehrstuhl für Wirtschaftsinformatik der Universität Hohenheim 2002. 
[GaWa01] Galla, M.; Wagner, M.: Partnersuche im E-Business. In: Virtuelle Organisationen und Neue Medien 2001. Hrsg.: M. Engelien; D. Neumann, Josef Eul Verlag, Lohmar, Köln 2001, S. 19-40.

[Hase93] Hasebrook, J.: Krebs-Selbsthilfegruppen - Untersuchungen zu Bedarf, Funktionen und Wirksamkeit. In: Onkologie im psychosozialen Kontext Spektrum psychoonkologischer Forschung, zentrale Ergebnisse und klinische Bedeutung. Hrsg.: F. A. Muthny; Gunther Haag, Roland Asanger Verlag, Heidelberg 1993.

[Kim01] Kim, A.J.: Community Building - Strategien für den Aufbau erfolgreicher Web-Communities. Galileo Press, Bonn 2001.

$\left[\mathrm{Koch}^{+} 00\right]$ Koch, M.; Möslein, K.; Wagner, M.: Vertrauen und Reputation in Online-Anwendungen und virtuellen Gemeinschaften. In: Virtuelle Organisationen und Neue Medien 2000. Hrsg.: M. Engelien; D. Neumann, Josef Eul Verlag, Lohmar, Köln 2000, S. 69-84.

[LoBr98] Loscalzo, M.; Brintzenhofeszoc, K.: Brief Crisis Counseling. In: Psycho-Oncology. Hrsg.: J. C. Holland, Oxford University Press, New York 1998, S. 662-675.

[Luhm89] Luhmann, N.: Vertrauen. Ein Mechanismus der Reduktion von Komplexität. 3. Auflage, Enke Verlag, Stuttgart 1989.

[Moel02] Möller, A.: Qualitätskriterien von Gesundheitsinformationen. In: http://www.afgis.de/ueberafgis_qualitaetskriterien.php, zugegriffen am 20.11.2002.

[Pico $\left.{ }^{+} 01\right]$ Picot, A.; Reichwald, R.; Wigand, R.: Die grenzenlose Unternehmung - Information, Organisation und Management. 4. Auflage, Thomas Gabler Verlag, Wiesbaden 2001.

[PrGh01] Preece, J.; Ghozati, K.: Experiencing Empathy Online. In: The Internet and Health Communication: Experience and Expectations. Hrsg.: R.R. Rice; J.E. Katz, Sage Publications, Thousand Oaks 2001, S. 237-260.

[Ripp98] Ripperger, T.: Ökonomik des Vertrauens: Analyse eines Organisationsprinzips. Mohr Siebeck, Tübingen 1998.

[Schn00] Schneidermann, B.: Designing trust into online experiences. In: Communications of the ACM, Vol. 43 (2000) Nr. 12, S. 57-59.

[Wahr86] Wahrig, G.: Wahrig - Deutsches Wörterbuch. Bertelsmann Lexikon Verlag, Gütersloh, München 1986.

[Wolf00] Wolff, B.: Kalkül und Vertrauen. Zur Institutionalisierung von Moral in der Ökonomik - Manuskript zum Vortrag im Rahmen der Tagung "Vertrauen in Gesellschaft und Organisationen". Tutzing, 20. Mai 2000. 